\title{
SIGNIFICANCE OF AMBIENT CONDITIONS IN URANIUM ABSORPTION AND EMISSION FEATURES OF LASER ABLATION PLASMAS
}

\author{
P. J. Skrodzki ${ }^{1,3,4}$, N. P. Shah ${ }^{1,3}$, N. Taylor ${ }^{1}$, K. C. Hartig ${ }^{1,2}$, N. L. LaHaye ${ }^{1}$, B. E. Brumfield ${ }^{1}$, I. Jovanovic ${ }^{3}$, M. C. \\ Phillips ${ }^{1, *}$, S. S. Harilal ${ }^{1, *}$ \\ ${ }^{1}$ Pacific Northwest National Laboratory, Richland, WA 99352, U.S.A. \\ ${ }^{2}$ Department of Mechanical and Nuclear Engineering, The Pennsylvania State University, University Park, PA 16802, U.S.A \\ ${ }^{3}$ Department of Nuclear and Radiological Sciences, University of Michigan, Ann Arbor, MI 48109, U.S.A. \\ ${ }^{4}$ School of Nuclear Engineering, Purdue University, West Lafayette, IN 47906, U.S.A.
}

\begin{abstract}
This study employs laser ablation (LA) to investigate mechanisms for $\mathrm{U}$ optical signal variation under various environmental conditions during laser absorption spectroscopy (LAS) and optical emission spectroscopy (OES). Potential explored mechanisms for signal quenching related to ambient conditions include plasma chemistry (e.g., uranium oxide formation), ambient gas confinement effects, and other collisional interactions between plasma constituents and the ambient gas. LA-LAS studies show that the persistence of the $U$ ground state population is significantly reduced in the presence of air ambient compared to nitrogen. LA-OES yields congested spectra from which the U I $356.18 \mathrm{~nm}$ transition is prominent and serves as the basis for signal tracking. LA-OES signal and persistence vary negligibly between the test gases (air and $\mathrm{N}_{2}$ ), unlike the LA-LAS results. The plume hydrodynamic features and plume fundamental properties showed similar results in both air and nitrogen ambient. Investigation of $U$ oxide formation in the laser-produced plasma suggests that low $U$ concentration in a sample hinders consistent detection of UO molecular spectra.
\end{abstract}

Keywords: Laser ablation; Optical emission spectroscopy (OES); Laser absorption spectroscopy (LAS); Laser-induced breakdown spectroscopy (LIBS); Plasma chemistry; Plasma diagnostics

*Corresponding authors: M. Phillips (mark.phillips@pnnl.gov), S. Harilal (hari@pnnl.gov) 


\section{Introduction}

Laser ablation (LA) has a growing range of applications related to spectrometry, propulsion, micromachining, thin film deposition, etc. [1-4]. Popular LA spectrometric techniques include LA-inductively coupled plasma-mass spectrometry (LA-ICP-MS) [5-7], LA-laser absorption spectroscopy (LA-LAS) [2, 8-10], LA-optical emission spectroscopy (LA-OES, commonly called laser-induced breakdown spectroscopy or LIBS) [1, 11], and LA-laser induced fluorescence (LA-LIF) [12]. Such analytical techniques provide elemental and isotopic information about target materials. Arguably the most robust technique is LA-OES when considering its simplicity, practicality, near-instantaneous in-situ remote detection capability $[1,13,14]$, and portability [15]. LA-OES analyzes emission from the cooling laserproduced plasma (LPP). Optical emission spectra act as fingerprints for specific plasma constituents. However, inherent disadvantages exist with LA-OES such as higher limits of detection in comparison to the other spectrometric techniques $[16,17]$ and potentially detrimental matrix effects in multi-constituent targets [18]. Nevertheless, LA-OES is practical for numerous applications including nuclear forensics. LA-LAS measures the inverse phenomenon to LA-OES. A continuous wave (CW) probe laser is transmitted through the LPP, and plasma constituents absorb at a particular wavelength that corresponds to the excitation energy of a transition. Changing the wavelength of the probe laser allows an absorption spectrum to be measured. Compared to LA-OES, LA-LAS provides higher sensitivity and is capable of probing both ground and excited state populations in a laser-plasma system. Furthermore, since LA-LAS can probe ground state transitions, plasma fundamental information may be gathered at very late times $(\geq$ $100 \mu \mathrm{s}$ ) during the plasma evolution when emission is too weak to detect because of lower plasma temperatures [2].

Nuclear forensic sciences require innovative approaches for the purpose of industrial safeguard certification, nuclear fuel prospecting, nuclear fuel cycle analysis, non-proliferation, treaty verification, etc. Additionally, nuclear forensic techniques must be safe, portable, and practical. LA-OES (or LIBS) meets most of these criteria (rapid analysis, fieldable, no sample preparation), albeit with some challenges with regard to characterization and identification of nuclear materials (e.g., U). The electronic complexity of high-Z elements, or elements with high partition functions, such as $U$, generates congested spectra in which specific emission features are challenging to discern without high-resolution spectroscopic equipment $[2,3,5,15,19,20]$. Furthermore, samples may only contain trace amounts of $U$ in a multiconstituent matrix, allowing emission features of more abundant target constituents to obfuscate those of $\mathrm{U}[13,18]$. Consequently, further investigation into the practicality of LA-OES for usage in the nuclear forensics field is paramount.

Limited studies exist on the practicality of LA-OES for U identification $[3,13,16,19]$. Notably, LAOES proves practical in detecting isotopic shifts of elements including $U$ [21-27]. Reported $U$ detection limits employing LA-OES are on the order of parts per million (ppm) [20, 28, 29]. However, previous investigations also suggest that the $\mathrm{U}$ signal varies significantly with ambient conditions using different LA spectrometric techniques [30]. Moreover, U emission as well as absorption lifetimes or persistence vary significantly with respect to environment, i.e., the nature of the ambient gas and system pressure [2, 13].

Typically, all LA-based standoff detection tools are operated in air ambient; however, the presence of oxygen in the air can alter the plasma chemistry and hence the emission/absorption features. This study investigates absorption and emission spectroscopy of $U$ species in a laser-plasma system and explores various mechanisms leading to $U$ signal quenching. The relevant plasma diagnostic techniques include LA-LAS, LA-OES, optical time-of-flight (OTOF) emission spectroscopy, and shadowgraphy. Ablation and plasma formation occurs in a vacuum chamber with varying ambient conditions. Plasma features within two different gases $\left(\mathrm{N}_{2}\right.$ and air) at different pressures are investigated. Plasma chemistry (oxide formation), plasma confinement effects, and changes in plasma fundamental properties are investigated as plausible mechanisms for variation in U signal in emission and absorption. 


\section{Experimental details}

A schematic of the experimental setup for LA-LAS, LA-OES, and OTOF is given in Figure 1, while an example schematic for shadowgraphy can be found in ref. [31]. For LA, $1064 \mathrm{~nm}$ pulses from a Qswitched Nd:YAG laser are used. The pulse energy is varied using a half-wave plate and cube polarizer. The laser beam is focused by a plano-convex lens onto a borosilicate glass sample containing $1.3 \%$ depleted $\mathrm{UO}_{2}$ by weight. The target $(3 \mathrm{~mm}$ thickness and $25 \mathrm{~mm}$ diameter) is mounted on a motorized translation stage which is moved to avoid target cratering effects. Experiments are performed at different background pressures. Two different gases $\left(\mathrm{N}_{2}\right.$ and air) are used to study confinement and plasma chemistry.
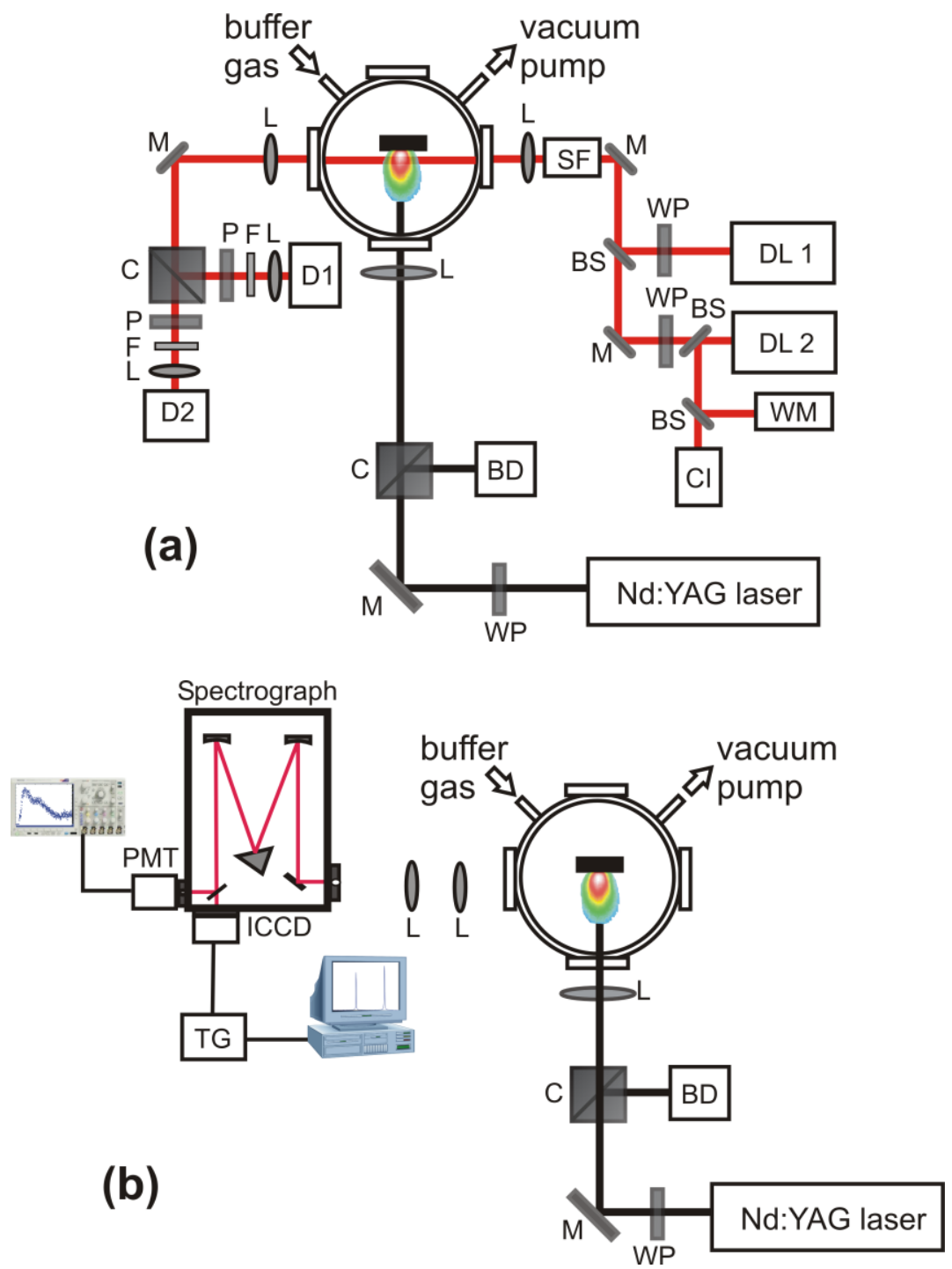

Figure 1. Experimental schematic for (a) LA-LAS and (b) LA-OES (BD: beam dump, BS: beam splitter, C: polarizing beam splitter cube, CI: confocal interferometer, D: detector, DL: diode laser, F: filter, ICCD: intensified charge-coupled device, L: lens, M: mirror, P: polarizer, PMT: photomultiplier tube, SF: spatial filter, TG: timing generator, WM: wavemeter, WP: wave plate). 
Methods for $U$ detection in this study include LA-LAS, LA-OES, and OTOF. The LA-LAS measurements use a differential CW-tunable diode laser setup to probe the ${ }^{238} \mathrm{U}$ I $860.795 \mathrm{~nm}$ transition. Two spatially overlapped and co-propagating diode lasers with linewidths of approximately $5 \mathrm{MHz}$ pass through the plasma, with the reference laser (DL1 in Fig. 1(a)) being a distributed feedback (DFB) diode laser that remains fixed at a wavelength $\sim 20 \mathrm{GHz}$ detuned from the transition and the probe laser (DL2 in Fig. 1(a)) is an external cavity diode laser (ECDL) that scans its wavelength over the desired atomic transition. A scanning confocal interferometer ensures the probe laser remains single-mode while scanning the wavelength and acquiring spectra. The wavelength of the probe laser is measured using a wavemeter with $10 \mathrm{MHz}$ resolution. The reference and probe beams were polarized orthogonally and combined using a polarizing beam splitter cube and pass through a spatial filter to improve the beam quality. The beams were then focused using a $25 \mathrm{~cm}$ lens and pass through the plasma $0.6 \mathrm{~mm}$ above the target surface, yielding a focused spot size in the LPP of $\sim 200 \mu \mathrm{m}$. After propagation through the plasma, the beams are separated using a polarizing beam splitter cube and detected with two silicon photodiodes with gain of $+50 \mathrm{mV} / \mu \mathrm{A}$ and bandwidth of $3 \mathrm{MHz}$. This signal is transferred into a 16-bit ADC at a $1 \mu \mathrm{s}$ sampling rate. The reference beam was used to remove noise from particle ejection and beam steering within the plasma. More information regarding experimental details and data processing can be found in Taylor and Phillips [2].

Emission spectroscopy involves imaging the plasma plume onto the slit of a $0.5 \mathrm{~m}$ spectrograph with an intensified CCD (ICCD) camera and a photomultiplier tube (PMT) occupying the two exit slits. The spectrograph has a 3-grating capability $(300 \mathrm{~g} / \mathrm{mm}, 1200 \mathrm{~g} / \mathrm{mm}$, and $2400 \mathrm{~g} / \mathrm{mm})$, with a maximum resolution of $\sim 0.03 \mathrm{~nm}$ when using the $2400 \mathrm{~g} / \mathrm{mm}$ grating. The slit width during the experiment is kept constant at $20 \mu \mathrm{m}$. Spectroscopic data is collected at a distance $1.5 \mathrm{~mm}$ above the target surface. OTOF data is obtained using a monochromator-PMT configuration, and signal features are recorded using a 1 $\mathrm{GHz}$ bandwidth oscilloscope. Additionally, emission spectroscopy of a U pure metal target was carried out at Penn State University under similar LA conditions (laser fluence $\sim 80 \mathrm{~J} / \mathrm{cm}^{2}$, air at atmospheric pressure).

Focused shadowgraphy tracks shockwave propagation in each of the two gases at $\sim 700$ Torr pressure. The experimental parameters were similar to the ones used for emission spectroscopy. In this setup, a relay lens images the plasma plume onto a CMOS detector (1280x960 pixels) [32]. $532 \mathrm{~nm}, 4 \mathrm{~ns}$ pulses from an Nd:YAG laser provide backlight. A timing generator synchronizes the arrival of the backlight pulse to that of the ablation laser pulse.

\section{Results \& analysis}

LA for analytical techniques has a vast parametric space. Absorption and emission signals are transient in nature, vary with location in LPP, and vary with ablation parameters (i.e. ablation wavelength, nature of the ambient gas, ambient gas pressure) [13]. LA-LAS, despite requiring a more complex experimental setup, is more sensitive than standard LIBS and is able to measure ground state populations, which is useful in understanding the properties of the plasma at later times. LA-OES, on the other hand, has lower sensitivity and exhibits congested spectra for high- $Z$ elements, but is a more simple and robust experimental technique. Moreover, LA-OES allows for efficient and coarse spectral analysis that is practical in identifying molecules [32]. OTOF provides a well-resolved transient signal for a single emission transition, effectively providing details about emission as a function of lifetime of the particular species [30]. Finally, shadowgraphy is an imaging technique used commonly for tracking particle formation and shockwave phenomenology in the LPP [32].

\subsection{Laser absorption spectroscopy}

In LA-LAS, a probe beam is transmitted through the ablation plume to excite the analyte of interest and is a useful analytical method for isotope analysis [10,33]. A laser fluence of $\sim 50 \mathrm{~J} / \mathrm{cm}^{2}$ and ablation 
spot size of $\sim 200 \mu \mathrm{m}$ was used for LA-LAS studies of the $860.795 \mathrm{~nm}$ transition $\left(\mathrm{f}^{3} \mathrm{ds}^{2}{ }^{5} \mathrm{~L}_{6} \rightarrow \mathrm{f}^{2} \mathrm{~d}^{2} \mathrm{~s}^{2}{ }^{5} \mathrm{~K}_{5}\right.$, $\left.0-11613.977 \mathrm{~cm}^{-1}, g A=1.3 \times 10^{7} / \mathrm{s}\right)$. In this work, we define the absorption, $A(t)$, as

$$
A(t)=-\ln \left(\frac{I(t)}{I_{o}}\right)
$$

where $I(t)$ is the intensity of the probe laser incident on the detector at time $t$ and $I_{o}$ is the intensity of the probe laser prior to the arrival of the ablation pulse. Figure 2 shows representative absorption spectra in 100 and 760 Torr $\mathrm{N}_{2}$ and air, $5 \mu$ s after ablation; the spectra were then fitted with a Voigt function to obtain spectral details (area under the peak, full width half maximum (FWHM), etc.). As observed previously, the $U$ absorption linewidth is very narrow $(<5 \mathrm{pm})$ at this time in the plasma evolution [2] and is smaller than the $12 \mathrm{pm}{ }^{235} \mathrm{U}-{ }^{238} \mathrm{U}$ isotope shift for this transition. Since the FWHM was approximately the same for the two gases, details are not provided here.

Figures 3 and 4 illustrate how the U signal (peak area) and persistence differ significantly whether the background gas is air or $\mathrm{N}_{2}$ at atmospheric pressure or 100 Torr. The area under the peak of the Voigt fit (shown as a solid line in Figure 2) is plotted versus time. The significantly reduced absorption and atomic lifetime in air versus nitrogen environment appears to indicate that chemical reactions with the oxygen in air are important. The formation of oxides would remove ground state atomic uranium from the system, thereby reducing the absorption signal from the $860.795 \mathrm{~nm}$ transition. The LA-LAS results prompt further investigation into the potential mechanisms governing the plasma plume behavior as a function of ambient environment.
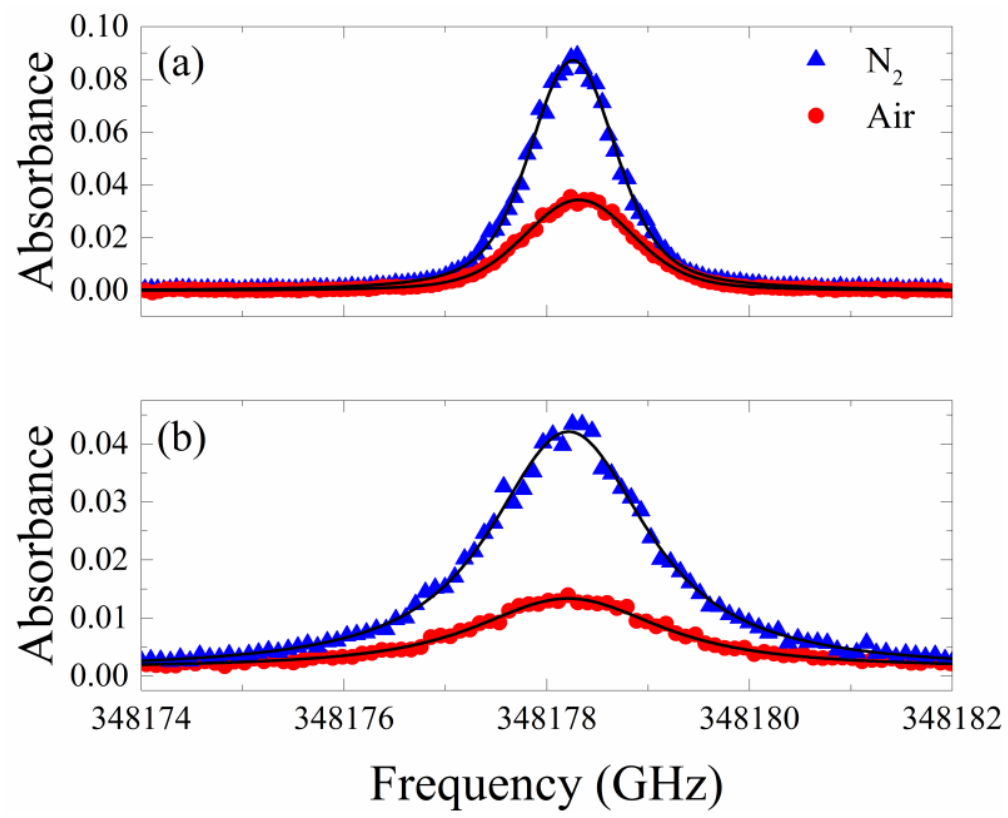

Figure 2. U I $860.795 \mathrm{~nm}$ absorption spectrum in (a) 100 Torr and (b) 760 Torr $\mathrm{N}_{2}$ and air, obtained $5 \mu$ s after ablation. The solid line represents a Voigt fit to the data points, which is a convolution of the Lorentzian and Gaussian line shapes. 


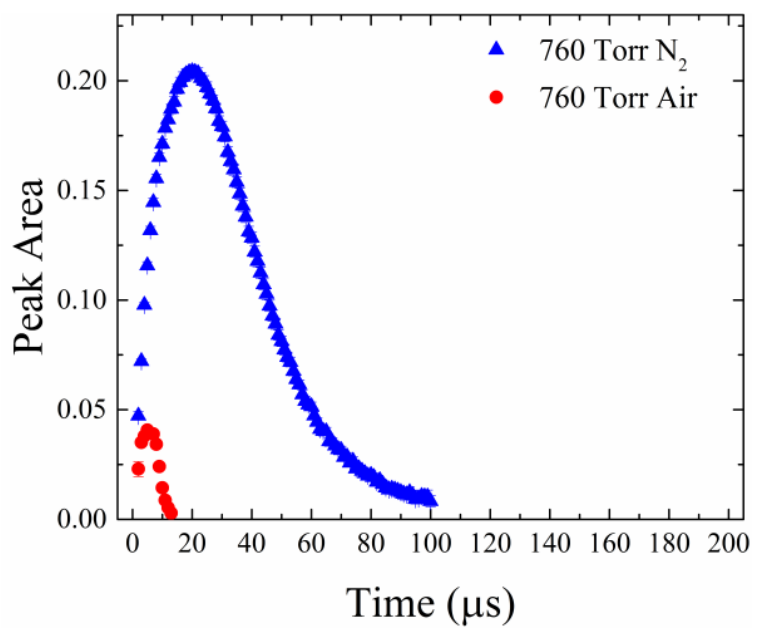

Figure 3. Temporal evolution of the U I $860.795 \mathrm{~nm}$ transition absorption peak area at 760 Torr $\mathrm{N}_{2}$ and air ambient.

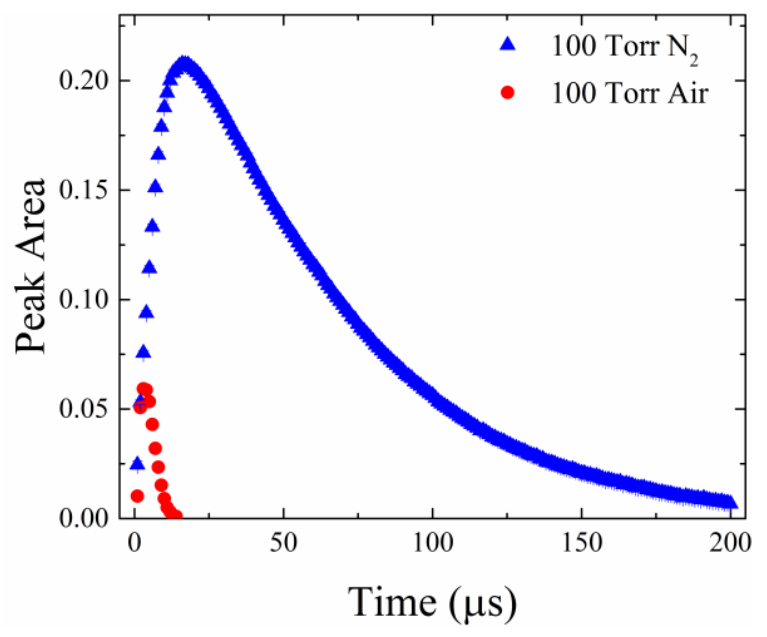

Figure 4. Temporal evolution of the U I $860.795 \mathrm{~nm}$ transition absorption peak area at 100 Torr $\mathrm{N}_{2}$ and air ambient.

An increase in ambient pressure leads to increased confinement of the plasma which in turn changes the morphology as well as fundamental parameters of the plasma. This will affect both the absorption and emission properties of plasma. The absorption lifetimes showed vast differences in 100 Torr and 760 Torr $\mathrm{N}_{2}$ ambient pressures $(\sim 137 \mu \mathrm{s}$ vs $\sim 80 \mu \mathrm{s})$, while the peak area (in this case, it is proportional to the number density in the ground state) showed more or less similar values. The higher confinement as the pressure increases may increase the total atomic number density; however, the ground state number density is depleted due to higher excitation temperature at early times. In addition, the path length for absorption is reduced as the pressure increases. The net result is that the maximum peak area does not change much with pressure. The changes in lifetime of peak area with $\mathrm{N}_{2}$ pressures could be due to similar reasons such as changes in plasma morphology/density, excitation temperature, and time evolution with changes in pressure. However, it has to be mentioned that it is difficult to draw definite conclusions on this topic because the plasma absorption was only probed at a single distance from the sample and the plasma morphology will be different in both space and time as the pressure changes. 


\subsection{Optical emission spectroscopy}

LA-LAS shows significant differences in $\mathrm{U}$ signal and persistence between air and $\mathrm{N}_{2}$ at identical pressures. The abundance of reactive species such as $\mathrm{O}_{2}$ in air may be responsible for quenching the $\mathrm{U}$ signal due to the formation of $U$ oxides, reducing the atomic $U$ concentration in the LPP [34]. Consequently, further investigation with emission spectroscopic methods yields probable mechanisms for the $U$ signal and persistence reduction between ambient gases. Emission features of $U$ as well as other sample constituents are compared between $\mathrm{N}_{2}$ and air at varying system pressures between 1 and 760 Torr. Figure 5 shows a typical emission spectrum between 325 and $370 \mathrm{~nm}$ for a U-containing sample for several system pressures, comparing $\mathrm{N}_{2}$ and air. A laser fluence of $20 \mathrm{~J} / \mathrm{cm}^{2}$ and spot size of $\sim 800 \mu \mathrm{m}$ were used for ablation for the OES studies.
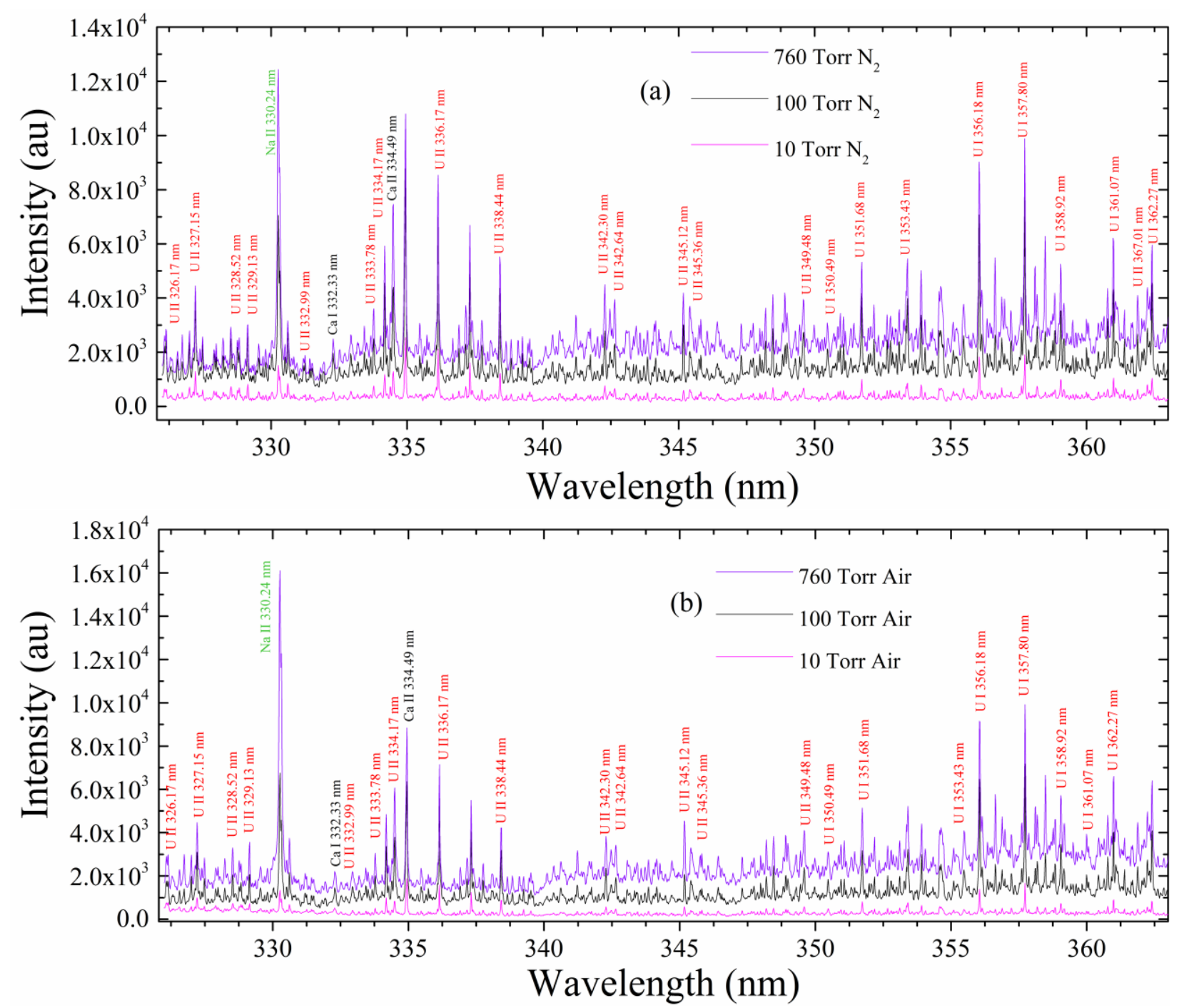

Figure 5. Spectra of various U I and U II emission lines (labeled in red) acquired using a glass sample among abundant matrix constituent features ( $\mathrm{Ca}$ - black; $\mathrm{Na}$ - green) at 10, 100, and 760 Torr compared between (a) $\mathrm{N}_{2}$ ambient and (b) air ambient. The corresponding acquisition information is as follows: $2 \mu$ s ICCD gate delay; $5 \mu \mathrm{s}$ integration period; viewing location $\sim 1.5 \mathrm{~mm}$ axial distance from target. Line assignments are based on the NIST database [35]. 
It is well known that the pressure and nature of the ambient environment affects the plasma emission properties greatly [30]. As Figure 5 shows, both intensity and background increase with increasing system pressure, although more gradually beyond 100 Torr. According to Chinni et al. [3], the presence of strong background emission seen in U plasmas could be due to overlapping of high density U lines. Notably, the best observed signal (comparing intensity to background) for the most prominent $U$ emission features, namely the U I $356.18 \mathrm{~nm}$ line among others, occurs at pressures $\mathbf{>} 10$ Torr pressure in each gas. Figure 6 shows time-integrated emission intensity of neutral $U$ species at an axial distance of $\sim 1.5 \mathrm{~mm}$ from the target surface as a function of pressure for both ambient gases $\left(\mathrm{N}_{2}\right.$ and air). Emission intensity increases as a function of pressure and plateaus or even decreases beyond 100 Torr. Unlike LA-LAS, only minor differences in intensity are observed between $\mathrm{N}_{2}$ and air.

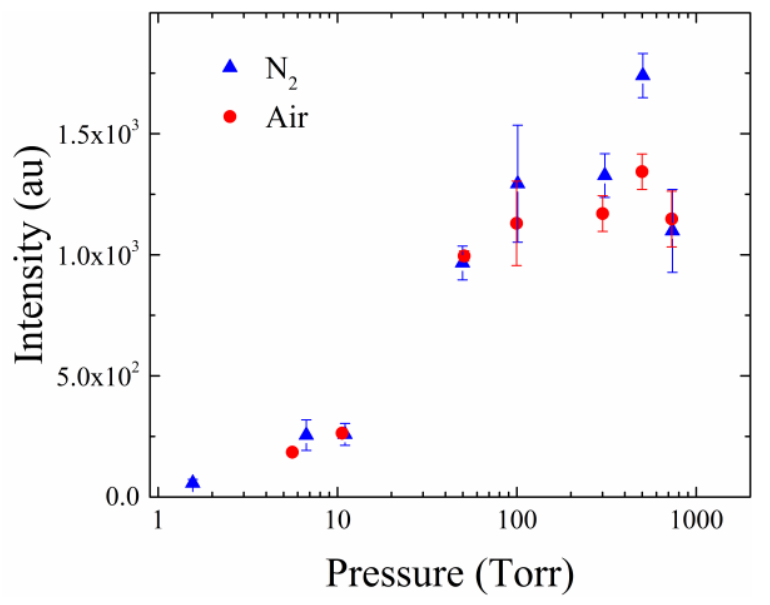

Figure 6. Time-integrated U I $356.18 \mathrm{~nm}$ emission signal as a function of system pressure for $\mathrm{N}_{2}$ and air.

Previous studies also reported significant changes in emission intensity with nature and pressure of the ambient environment [36, 37]. OTOF measurements are carried out for the U I transition at $356.18 \mathrm{~nm}$ under similar conditions depicted in Figure 6 to obtain persistence information. OTOF measurements of a constituent species in the plume at a specific point within the plasma provide arrival time (and hence velocity) and persistence of a line transition (Figure 7 inset). The persistence of $U$ transitions is estimated by measuring the time at which the intensity drops to $\mathrm{e}^{-2}$ from the OTOF peak; results are given in Figure 7. Persistence generally increases with increasing system pressure because the plasma expansion rate decreases logarithmically with increasing pressure. Hence emission is confined to a location axially closer to the target surface where plasma temperatures are typically greater than if the plasma is allowed to expand in vacuum-like conditions (or low pressures) [38-40]. Therefore, at an axial distance of $\sim 1.5 \mathrm{~mm}$ from the target surface, the emission persistence appears greater at higher pressures. 


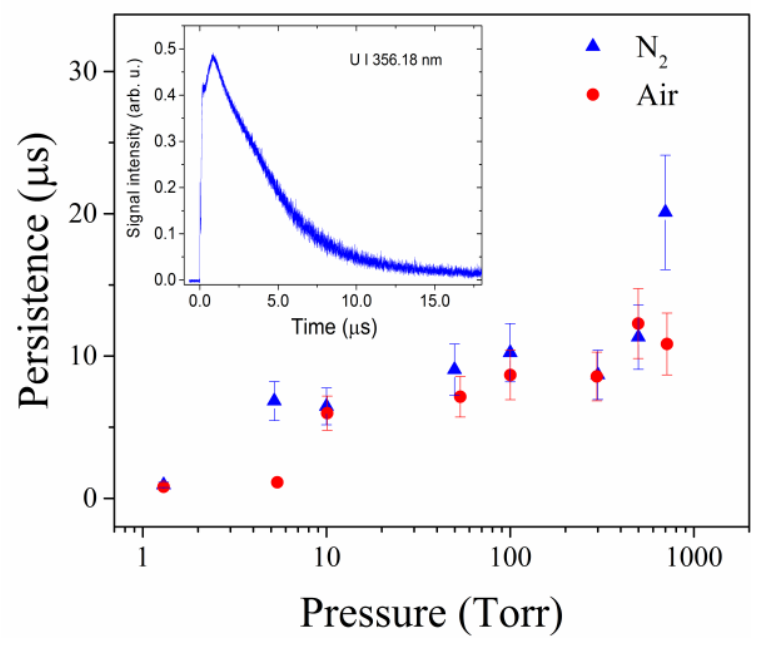

Figure 7. U I $356.18 \mathrm{~nm}$ persistence as a function of system pressure for $\mathrm{N}_{2}$ and air. Typical OTOF profile recorded at $\sim 100$ Torr air pressure is given in the inset.

In the OTOF measurements, the $\mathrm{U}$ species persist slightly longer in $\mathrm{N}_{2}$ versus air, but the difference is minor compared to the dramatic effect observed in LA-LAS. One reason for the small differences from OTOF could be that the emission persistence is significantly lower than the absorption persistence at all pressures. Absorbance signal corresponds to the number density of the ground state population, while emission intensity and persistence correspond to the number density of the excited level population. The time evolution of population density of a particular excited state is expected to be significantly faster than the evolution of a ground level population in the transient laser plasma system. Moreover, the selected U I lines for absorption and emission studies are resonance lines; hence the ground level population and its persistence will be higher compared to excited level populations, explaining the observed difference between absorption and emission signal longevities. The reduction in the absorption signal in air primarily occurs at times $\widetilde{>} 10 \mu \mathrm{s}$, so it may be that the OTOF measurement is not sensitive to this change since the emission signal is weak at these times.

\subsection{Investigation of $U$ oxide formation in $L P P$}

A plausible mechanism for $\mathrm{U}$ emission quenching in $\mathrm{N}_{2}$ and air involves the formation of $\mathrm{U}$ containing compounds, namely $U$ oxides or nitrides. Limited studies exist of spectroscopic data for $U$ nitrides [41] and U oxides [42-44]. However, the results from LA-LAS measurements discussed in Section 3.1. suggest that the rate of $U$ oxide formation is significantly greater than $U$ nitride formation because the peak area and persistence of U I absorption at $860.795 \mathrm{~nm}$ is significantly lower in air than in $\mathrm{N}_{2}$ at identical pressures. Moreover, the Gibbs free energies of the reaction for UN are greater than those for $\mathrm{UO}$ and $\mathrm{UO}_{2}$ under similar conditions [45]. Based on this information, the formation of $\mathrm{U}$ oxide is investigated as a possible mechanism to explain the quenching of the $\mathrm{U}$ atomic signal in the LA-LAS studies presented in Section 3.1. 


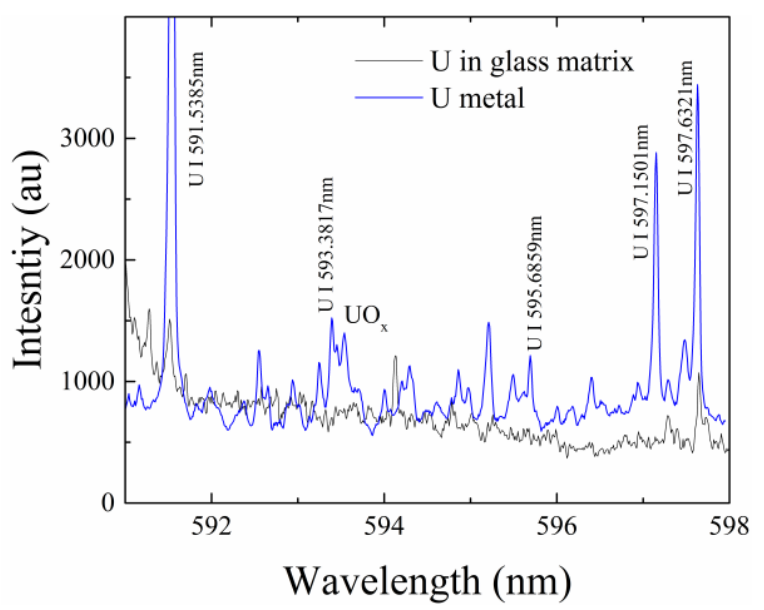

Figure 8: Spectral features recorded from $U$ in a glass matrix and a $U$ metal target. The spectra were captured with a gate delay of $10 \mu \mathrm{s}$ and gate width of $100 \mu \mathrm{s}$. The measurements were performed in air at atmospheric pressure.

To study uranium oxide formation, emission spectra over a $590-600 \mathrm{~nm}$ spectral range were recorded with the ambient air pressure at 4 or 750 Torr. The selection of this spectral range is based on reported uranium oxide electronic band centers from multiple literature sources [34, 42, 43]. A typical spectrum obtained from $U$ in a glass matrix is given in Figure 8. For comparison, the spectral features obtained from a $\mathrm{U}$ metal target (depleted) recorded under approximately similar experimental conditions (laser fluence $\sim 80 \mathrm{~J} / \mathrm{cm}^{2}$, air at atmospheric pressure) are also given in Figure 8 . The emission features from $\mathrm{U}$ metal show intense lines from U I (marked in the figure) along with an assigned $\mathrm{UO}_{\mathrm{x}}$ band centered around $593.6 \mathrm{~nm}$ [43]. However, no electronic band structures were observed that could be assigned to $\mathrm{UO}_{\mathrm{x}}$ in the emission spectra from the $\mathrm{U}$ in glass matrix. One plausible explanation for this lack of detection is that formation of larger $\mathrm{U}$ oxide $\left(\mathrm{U}_{\mathrm{x}} \mathrm{O}_{\mathrm{y}}\right)$ species is rapid and efficient when the number density of oxygen is present in a significant excess over the $\mathrm{U}$ number density in the plume. The efficient formation of $\mathrm{U}_{\mathrm{x}} \mathrm{O}_{\mathrm{y}}$ has been noted previously in low-pressure flow reactor studies of $\mathrm{U}$ atoms with $\mathrm{O}_{2}$ [42]. Based on theoretical kinetic modeling of $\mathrm{U}_{\mathrm{x}} \mathrm{O}_{\mathrm{y}}$ formation in the flow reactor study, the initial number density of $U$ needed to be 5 times larger than the $\mathrm{O}_{2}$ number density to optimize the concentration of UO at the exit of the flow reactor where it was to be measured using IR LAS [42]. Increasing the $\mathrm{O}_{2}$ concentration beyond the above ratio significantly reduced the formation of UO in favor of larger oxide species. While the experimental conditions in an LPP are different from those of the low-pressure flow reactor, there has been one reported detection of UO emission from a LIBS study that supports the assertion that an excess of $\mathrm{O}_{2}$ significantly reduces the number density of $\mathrm{UO}$ [34]. In that study, $\mathrm{U}$ metal was ablated in air and pure $\mathrm{O}_{2}$, and when the ablation was performed in a pure $\mathrm{O}_{2}$ ambient background there was a significant reduction in the UO emission intensity compared to in air [34]. Unlike the LIBS study employing $\mathrm{U}$ metal, where an emission feature of $\mathrm{UO}_{\mathrm{x}}$ was evident, the U-doped glass target contains $\sim 1 \% \mathrm{U}$ by mass. Hence, the absence of UO emission from the U-doped target could be due to use of a glass target where the abundance of $U$ is 2 orders of magnitude lower than in a pure $U$ metal target. As a consequence, a significant excess of $\mathrm{O}_{2}$ is present that leads to rapid formation of $\mathrm{U}_{\mathrm{x}} \mathrm{O}_{\mathrm{y}}$ and depletes the UO concentration to the point that its emission is not detectable in the current experiment. This explanation is also consistent with the LA-LAS measurements, which measure the decrease of atomic $\mathrm{U}$ in air from all chemical reactions and is not dependent on the particular oxide formed.

\subsection{Plume confinement investigated through shadowgraphy}

To address the difference in absorption signals between $\mathrm{N}_{2}$ and air, we have evaluated the confinement of the plasma plumes under air and nitrogen gas environments using shadowgraphy. The 
interaction between a plume and the ambient gas is a complex, dynamic process involving deceleration, attenuation, and thermalization of the ablated species. Previous studies varying system pressures and expansion durations demonstrate that the plume-ambient interaction is characterized by different propagation phases accompanied by plume oscillations with increasing ambient pressure [40]. Moreover, the nature of the ambient gas dictates plume dynamics through the aforementioned chemical interaction or even collisional interaction. Our recent results highlight that plume hydrodynamics mediate molecular formation in laser ablation plumes [32]. Mass and density of the ambient gas influence momentum transfer, LPP heat dissipation, etc. Hence we investigate hydrodynamic expansion features through shockwave propagation of U-containing LPP. Figure 9 provides information related to shockwave propagation tracked through shadowgraphy in $\mathrm{N}_{2}$ and air at $\sim 700$ Torr. A laser fluence of $20 \mathrm{~J} / \mathrm{cm}^{2}$ and spot size of $\sim 800 \mu \mathrm{m}$ were used for ablation.

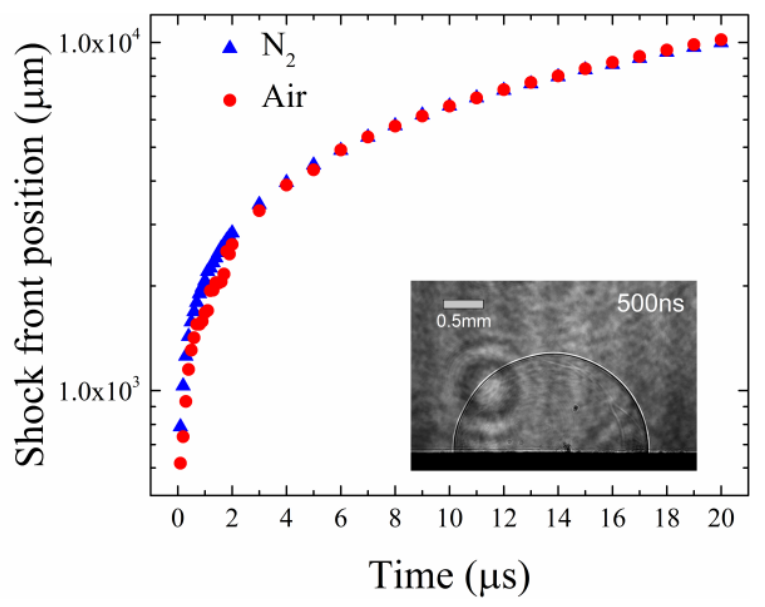

Figure 9. Transient shockwave propagation distance obtained using shadowgraphy tracking techniques in $\mathrm{N}_{2}$ and air. A representative shadowgram image recorded $500 \mathrm{~ns}$ after the onset of plasma generation in air ambient is given in the inset.

According to Figure 9, negligible differences exist in shockwave propagation throughout the first 20 $\mu$ s of plasma/shock evolution between the two ambient gases. Distance and speed trends are consistent with those reported by Hough et al. [26] using $\mathrm{O}_{2}$ as ambient gas. Notably, the shockwaves persist beyond a period of $20 \mu \mathrm{s}$ after ablation; however, camera chip size and image magnification impose limits on tracking beyond this point, as the shockwave travels outside the frame. Despite these limitations, shockwave propagation at these earlier times provides information about plasma confinement. Previous studies suggest that the plasma and shock propagate together for a few $\mu$ s before detachment, and beyond this point plasma imaging techniques are more representative of confinement effects [46]. Plasma confinement trends appear similar between gases at the earlier times presented, indicating confinement effects are similar between $\mathrm{N}_{2}$ and air. This can be understood considering the density of air and $\mathrm{N}_{2}$, which are approximately the same; this may also indicate that plasma confinement plays a minor role in the observed absorption signal and lifetime discrepancy. Consequently, confinement effects are not responsible for the observed differences in quenching $\mathrm{U}$ emission features between the two gases.

\subsection{Analysis of plasma fundamental properties}

Emission and absorption features of plasmas strongly depend on plasma fundamental properties, namely electron density and excitation temperature. The temporal evolution of density and temperature are measured in order to estimate their degree of influence on $\mathrm{U}$ signal and persistence varying between ambient gases. Temperature measurements are carried out by assuming the plasma is in local 
thermodynamic equilibrium (LTE) and employing the Boltzmann plotting method [47], while electron density is calculated through analysis of the linewidth of the Ca I $315.88 \mathrm{~nm}$ transition characterizing the Stark broadening phenomenon. The impact parameter of the selected line is obtained from Puric et al. [48].

Figure 10 shows time-resolved electron densities obtained for the various gases at 100 and 760 Torr system pressures. The relative uncertainties of the electron density measurement were estimated to be less than $10 \%$ based on the error in the fitting of the experimental data. Stark broadening related to internal plume pressures and densities is similar in both gases.
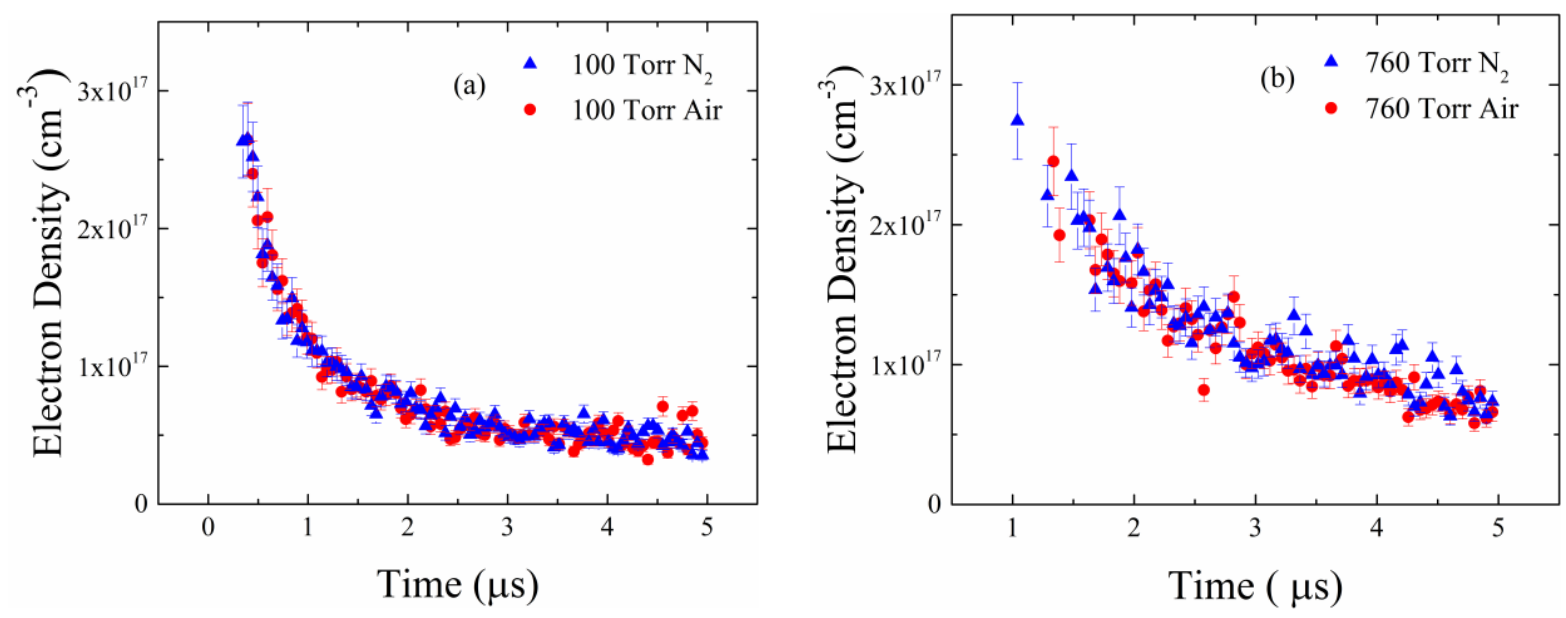

Figure 10. Temporal evolution of electron density for $\mathrm{N}_{2}$ and air with system pressures of (a) 100 and (b) 760 Torr at $\sim 1.5 \mathrm{~mm}$ axial distance from the uranium-containing glass target surface.

Figure 11 shows differences in time-resolved plasma excitation temperatures between $\mathrm{N}_{2}$ and air at 100 and 760 Torr system pressures. Electron temperatures are estimated by applying the Boltzmann plotting technique [47], comparing intensities of Ca II 315.89, 317.93, 370.6, 373.69, 393.36, and 396.85 $\mathrm{nm}$ transitions. The relative uncertainty of the electron temperature measurement was estimated to be $\sim 10 \%$. The temporal evolution of measured electron temperature values showed similar behavior regardless of the nature of the ambient gas used. These results are consistent with recent reports [49, 50]. However, a comparison of time-resolved measurements of temperatures and electron densities of laserproduced Fe plasmas generated in air, argon, and helium at atmospheric pressures by Aguilera and Aragon [51] showed that higher temperatures and electron densities were obtained in argon, and lower ones are found in helium. This indicates that the influence of type and pressure of the ambient gas on plasma properties is strongly related to experimental conditions as well as target physical properties. However, such a difference is not noticeable in the present experiment using air and nitrogen as ambient gases. Moreover, the measured temperature and electron density represent their evolution at a certain location in the LPP system $(\sim 1.5 \mathrm{~mm})$ and are not a representation of the entire plasma system. 

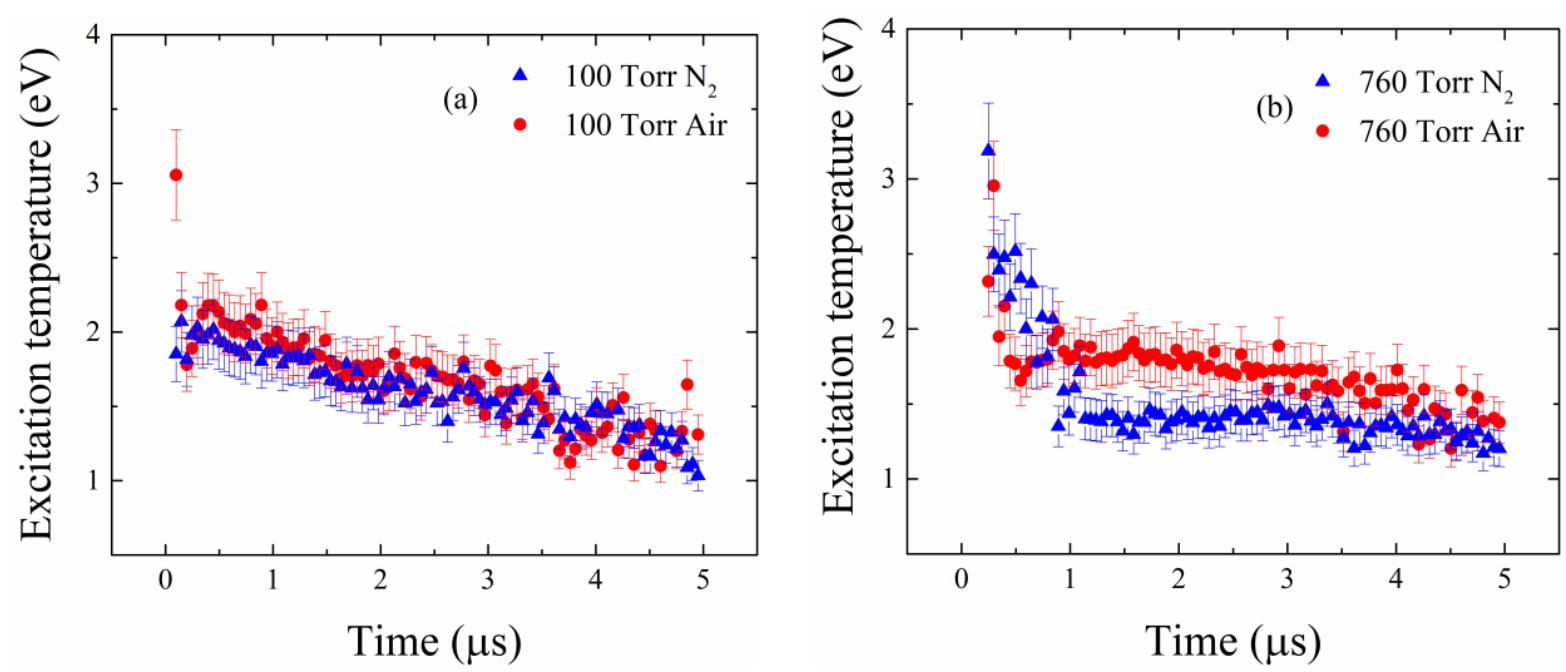

Figure 11. Time-resolved excitation temperatures estimated through the Boltzmann plotting method, comparing Ca II 315.89, 317.93, 370.6, 373.69, 393.36, and $396.85 \mathrm{~nm}$ transitions in $\mathrm{N}_{2}$ and air at (a) 100 and (b) 760 Torr pressures at $\sim 1.5 \mathrm{~mm}$ axial distance from the uranium-containing glass target surface.

A comparison between the excited state (emission) and ground state (absorption) population is of interest for a laser plasma system under different ambient environments. LA-LAS showed significant differences in persistence of $\mathrm{U}$ species in the plasma, where $\mathrm{N}_{2}$ ambient provided higher persistence compared to air. However, LA-OES of $U$ transitions showed only minor variation in persistence between air and $\mathrm{N}_{2}$. Emission persistence is found to be significantly lower compared to the absorption signal persistence in $\mathrm{N}_{2}$ ambient; however, it is similar under air ambient. The recorded persistence for ground state population for $\mathrm{U}$ I in $\mathrm{N}_{2}$ and air are $\sim 137 \mu$ s and $\sim 10 \mu \mathrm{s}$ at 100 Torr pressure, respectively, and $\sim 80$ $\mu \mathrm{s}$ and $\sim 10 \mu \mathrm{s}$ at 760 Torr pressure, respectively. The corresponding emission persistence is $\sim 10 \mu \mathrm{s}$ regardless of the nature of the ambient at 100 and 760 Torr pressure levels.

Comparing LAS and OES techniques, one has to consider the differences between absorption and emission spectroscopy. In the absorption spectroscopy done in this work, the atoms are initially in the ground state and absorb the probe beam photons, causing excitation and a drop in the intensity of the passing beam. Since LAS probes the population of the ground state of an electronic transition, an absorption signal can be obtained even at lower temperatures and/or at very late times of plasma evolution (U transition in $\mathrm{N}_{2}$ ambient persisted for $\sim 100 \mu \mathrm{s}$ ). In emission spectroscopy, the transition occurs from an excited state to a lower state. Emission intensity is governed mainly by the spontaneous emission lifetime of the transition as well as the temperature and density of the plasma system. Typically to see emission, higher plasma temperatures are necessary to excite the atoms to the upper energy level, and hence the emission signal from the various species is short-lived compared to absorption. Moreover, it has to be considered that in a transient system like a laser plasma at later times of its evolution, the population density of a particular excited state is significantly lower compared to the ground level population, considering numerous transitions share the same lower level state.

The plume hydrodynamic features and plume fundamental properties showed somewhat similar results in air and nitrogen ambient. Although this is consistent with the observed similarity in emission features in air and $\mathrm{N}_{2}$ (intensity, persistence, etc.), these results are not adequate to explain the significant differences observed in the persistence of ground level populations of $U$ species. The uranium oxide emission studies also do not provide evidence for significant oxide formation with targets containing $U$ as a trace element, though strong uranium oxide emission was observed from ablation of the pure U metal target. The lack of oxide emission from the U-containing glass target could also be due to strong background emission obscuring potentially weak molecular band features. The chemistry leading to uranium oxide formation is not fully understood at this time and requires further study. 


\section{Conclusions}

In this article, a systematic study has been carried out on absorption and emission features of $U$ in an LPP under various ambient conditions. Investigation of $U$ behavior in an LPP employing LA-LAS revealed a significant reduction in persistence of atomic ground state transition between ambient air and $\mathrm{N}_{2}$ environments. The significantly reduced absorption and atomic lifetime in air versus nitrogen environment appears to indicate that chemical reactions with the oxygen in air are important. Subsequently, LA-OES experiments examining the effects of ambient environment on $U$ signal provide further details regarding quenching mechanisms. Emission features of $U$ as well as other sample constituents are compared between $\mathrm{N}_{2}$ and air at varying system pressures between 1 and 760 Torr. LAOES signal and persistence show minor differences between the test gases (air and $\mathrm{N}_{2}$ ), dissimilar to the large differences obtained from LA-LAS.

Shadowgraphic studies have been carried out to explore the differences in plume hydrodynamics with changes in the nature of the ambient gas and its role on emission features. However, negligible differences exist in shockwave propagation throughout the first $20 \mu \mathrm{s}$ of plasma/shock evolution between the two ambient gas cases. Ultimately, analysis of plasma fundamental properties, electron density and excitation temperature implies that plasma-ambient collisional interactions affect plasma cooling and hence signals of species within the LPP. The excitation temperature showed insignificant differences between the different gases. However, it should be emphasized that the measured temperature and density represent its evolution at a certain location in the LPP system $(\sim 1.5 \mathrm{~mm})$ and it is not representative of the entire plasma system.

An investigation into uranium oxide emission features revealed no band structures from $\mathrm{U}$ in a glass matrix; however, strong emission from uranium oxide bands were seen with a $U$ metal target. Previous studies have indicated that the number density of $\mathrm{U}$ needs to be roughly equivalent to the $\mathrm{O}_{2}$ number density to optimize the concentration of UO [42]. Hence, the absence of uranium oxide emission bands from plasma containing $U$ as a trace could be due to the low concentration of $U$ in the plasma. However, the spectral features from the U-containing target showed strong background emission, and any weak emission features from molecular bands may be difficult to discern. The chemistry leading to the formation of uranium oxides and compounds is not fully understood yet, and further study in this direction is necessary.

The large variation in ground state populations of $\mathrm{U}$ I transition in air and $\mathrm{N}_{2}$ seen during LA-LAS studies could be caused by plasma chemistry even though $U$ emission features did not give any conclusive evidence. Since LAS is probing the population of the ground state of an electronic transition an absorption signal can be obtained even at lower temperatures and/or at very late times of plasma evolution. In contrast, the emission from the plasma system persists when the temperature of the system is capable of exciting the atoms to the upper energy levels and hence emission is short lived. Further timeand space-resolved studies are necessary to explain the discrepancy between the persistence of U I species observed with LAS and OES. Moreover, the $U$ in glass matrix already contains a significant amount of oxygen atoms, and the reason why the quenching mechanism is not applicable in $\mathrm{N}_{2}$ environment compared to air ambient also needs further study. The present studies also highlight the importance of using multiple plasma diagnostic tools to better understand the finer aspects of transient plasma-ambient interaction.

\section{Acknowledgements}

This work is supported in part by the DOE/NNSA Office of Nonproliferation and Verification Research and Development (NA-22) and the Laboratory Directed Research and Development (LDRD) Program of Pacific Northwest National Laboratory. Pacific Northwest National Laboratory is operated for the U.S. DOE by the Battelle Memorial Institute under Contract No. DEAC05-76RLO1830. P. J. Skrodzki and N. P. Shah would like to acknowledge support and funding from Summer Undergraduate Laboratory Internship program sponsored by the U.S. DOE. This work was funded in part by the Consortium for Verification Technology under U.S. Department of Energy National Nuclear Security Administration Award Number DE-NA0002534 and by the U.S. Department of Homeland Security under Grant Award Number 2012.05 DN-130-NF0001. The views and conclusions 
contained in this document are those of the authors and should not be interpreted as representing the official policies, either expressed or implied, of the U.S. Department of Homeland Security.

\section{References}

[1] D. Hahn, N. Omenetto, Laser-Induced Breakdown Spectroscopy (LIBS), Part II: Review of Instrumental and Methodological Approaches to Material Analysis and Applications to Different Fields, Applied Spectroscopy, 66 (2012) 347-419.

[2] N.R. Taylor, M.C. Phillips, Differential laser absorption spectroscopy of uranium in an atmospheric pressure laser-induced plasma, Optics Letters, 39 (2014) 594-597.

[3] R.C. Chinni, D.A. Cremers, L.J. Radziemski, M. Bostian, C. Navarro-Northrup, Detection of Uranium Using Laser-Induced Breakdown Spectroscopy, Applied Spectroscopy, 63 (2009) 1238-1250.

[4] N. LaHaye, M.C. Phillips, A. Duffin, G. Eiden, S.S. Harilal, The influence of ns- and fs-LA plume local conditions on the performance of a combined LIBS/LA-ICP-MS sensor Journal of Analytical Atomic Spectrometry, 31 (2016) 515-522

[5] S. Boulyga, S. Konegger-Kappel, S. Richter, L. Sangely, Mass spectrometric analysis for nuclear safeguards, Journal of Analytical Atomic Spectrometry, 30 (2015) 1469-1489.

[6] N.L. LaHaye, P.K. Diwakar, J. Kurian, L. Alff, S.S. Harilal, Precise stoichiometric analysis of thin films using fs-LA-ICPMS, Scientific Reports, 5 (2015) 13121.

[7] D. Gunther, B. Hattendorf, Solid sample analysis using laser ablation inductively coupled plasma mass spectrometry, TracTrends in Analytical Chemistry, 24 (2005) 255-265.

[8] H. Liu, A. Quentmeier, K. NIemax, Diode laser absoption measurement of uranium isotope ratios in solid samples using laser ablation, Spectrochim. Acta Part B, 57 (2002) 1611-1623.

[9] B.A. Bushaw, M.L. Alexander, Investigation of laser ablation plume dynamics by high-resolution time-resolved atomic absorption spectroscopy, Applied Surface Science, 127 (1998) 935-940.

[10] M. Miyabe, M. Oba, H. Iimura, K. Akaoka, Y. Maruyama, H. Ohba, M. Tampo, I. Wakaida, Absorption spectroscopy of uranium plasma for remote isotope analysis of next-generation nuclear fuel, Applied Physics a-Materials Science \& Processing, $112(2013) 87-92$.

[11] S. Musazzi, U. Perini, Laser-Induced Breakdown Spectroscopy - Fundamentals and Applications Springer Series in Optical Sciences, Vol 182, 2014.

[12] S.S. Harilal, N.L. LaHaye, M.C. Phillips, Two-dimensional fluorescence spectroscopy of laser-produced plasmas, Optics Letters, 41 (2016) 3547-3550.

[13] N. LaHaye, S. Harilal, P. Diwakar, A. Hassanein, Persistence of uranium emission in laser-produced plasmas, Journal of Applied Physics, 115 (2014) 163301.

[14] J. Gottfried, F. De Lucia, C. Munson, A. Miziolek, Double-pulse standoff laser-induced breakdown spectroscopy for versatile hazardous materials detection, Spectrochimica Acta Part B-Atomic Spectroscopy, 62 (2007) 1405-1411.

[15] J. Goujon, A. Giakoumaki, V. Pinon, O. Musset, D. Anglos, E. Georgiou, J. Boquillon, A compact and portable laserinduced breakdown spectroscopy instrument for single and double pulse applications, Spectrochimica Acta Part B-Atomic Spectroscopy, 63 (2008) 1091-1096.

[16] N. Wogman, Prospects for the introduction of wide area monitoring using environmental sampling for proliferation detection, Journal of Radioanalytical and Nuclear Chemistry, 296 (2013) 1071-1077.

[17] J. Becker, Inductively coupled plasma mass spectrometry (ICP-MS) and laser ablation ICP-MS for isotope analysis of longlived radionuclides, International Journal of Mass Spectrometry, 242 (2005) 183-195.

[18] P.J. Skrodzki, J.R. Becker, P.K. Diwakar, S.S. Harilal, A. Hassanein, A comparative study of single-pulse and double-pulse LIBS with U-containing samples, Applied Spectroscopy, 70 (2016) 467-473.

[19] W. DeGraffenreid, C.J. Sansonetti, Reference lines in the optogalvanic spectra of uranium and thorium over the wavelength range 694-755 nm, J. Opt. Soc. Am. B, 19 (2002) 1711-1715.

[20] J.E. Barefield, E.J. Judge, K.R. Campbell, J.P. Colgan, D.P. Kilcrease, H.M. Johns, R.C. Wiens, R.E. McInroy, R.K. Martinez, S.M. Clegg, Analysis of geological materials containing uranium using laser-induced breakdown spectroscopy (LIBS), Spectrochimica Acta Part B: Atomic Spectroscopy, 120 (2016) 1-8.

[21] D.A. Cremers, A. Beddingfield, R. Smithwick, R.C. Chinni, C.R. Jones, B. Beardsley, L. Karch, Monitoring Uranium, Hydrogen, and Lithium and Their Isotopes Using a Compact Laser-Induced Breakdown Spctroscopy (LIBS) Probe and HighResolution Spectrometer, Applied Spectroscopy, 66 (2012) 250-261.

[22] W. Pietsch, A. Petit, A. Briand, Isotope ratio determination of uranium by optical emission spectroscopy on a laser-produced plasma - basic investigations and analytical results, Spectrochimica Acta Part B-Atomic Spectroscopy, 53 (1998) $751-761$.

[23] F.R. Doucet, G. Lithgow, R. Kosierb, P. Bouchard, M. Sabsabi, Determination of isotope ratios using Laser-Induced Breakdown Spectroscopy in ambient air at atmospheric pressure for nuclear forensics, Journal of Analytical Atomic Spectrometry, 26 (2011) 536-541.

[24] G.C.Y. Chan, X.L. Mao, I. Choi, A. Sarkar, O.P. Lam, D.K. Shuh, R.E. Russo, Multiple emission line analysis for improved isotopic determination of uranium - a computer simulation study, Spectrochimica Acta Part B-Atomic Spectroscopy, 89 (2013) 40-49.

[25] I. Choi, G.C.Y. Chan, X.L. Mao, D.L. Perry, R.E. Russo, Line Selection and Parameter Optimization for Trace Analysis of Uranium in Glass Matrices by Laser-Induced Breakdown Spectroscopy (LIBS), Applied Spectroscopy, 67 (2013) 1275-1284. 
[26] P. Ko, K.C. Hartig, J.P. McNutt, R.B.D. Schur, T.W. Jacomb-Hood, I. Jovanovic, Adaptive femtosecond laser-induced breakdown spectroscopy of uranium, Review of Scientific Instruments, 84 (2013).

[27] P.K. Morgan, J.R. Scott, I. Jovanovic, Hybrid interferometric/dispersive atomic spectroscopy of laser-induced uranium plasma, Spectrochim Acta B, 116 (2016) 58-62.

[28] E.C. Jung, D.H. Lee, J.-I. Yun, J.G. Kim, J.W. Yeon, K. Song, Quantitative determination of uranium and europium in glass matrix by laser-induced breakdown spectroscopy, Spectrochimica Acta Part B, 66 (2011) 761-764.

[29] Y.-S. Kim, B.-Y. Han, H.S. Shin, H.D. Kim, E.C. Jung, J.H. Jung, S.H. Na, Determination of uranium concentration in an ore sample using laser-induced breakdown spectroscopy, Spectrochimica Acta Part B, 74-75 (2012) 190-193.

[30] S. Harilal, P. Diwakar, N. LaHaye, M. Phillips, Spatio-temporal evolution of uranium emission in laser-produced plasmas, Spectrochimica Acta Part B: Atomic Spectroscopy, 111 (2015) 1-7.

[31] N. LaHaye, S. Harilal, P. Diwakar, A. Hassanein, Characterization of laser ablation sample introduction plasma plumes in fs-LA-ICP-MS, Journal of Analytical Atomic Spectrometry, 29 (2014) 2267-2274.

[32] S.S. Harilal, B.E. Brumfield, B. Cannon, M.C. Phillips, Shock wave mediated plasma chemistry of AlO formation in laser ablation plumes Analytical Chemistry, 88 (2016) 2296-2302.

[33] H. Liu, A. Quentmeier, K. Niemax, Diode laser absorption measurement of uranium isotope ratios in solid samples using laser ablation, Spectrochimica Acta Part B-Atomic Spectroscopy, 57 (2002) 1611-1623.

[34] D. Zhang, X. Ma, S. Wang, X. Zhu, Influence of ambient gas on laser-induced breakdown spectroscopy of uranium metal, Plasma Science and Technology, 17 (2015) 971-974.

[35] A. Kramida, Y. Ralchenko, J. Reader, N.A. Team, NIST Atomic Spectra Database, National Institute of Standards and Technology, http://physics.nist.gov/asd.

[36] N. Smijesh, R. Philip, Emission dynamics of an expanding ultrafast-laser produced Zn plasma under different ambient pressures, Journal of Applied Physics, 114 (2013) 093301.

[37] S. Sreedhar, E.N. Rao, G.M. Kumar, S.P. Tewari, S.V. Rao, Molecular formation dynamics of 5-nitro-2,4-dihydro-3H-1,2,4triazol-3-one, 1,3,5-trinitroperhydro-1,3,5-triazine, and 2,4,6-trinitrotoluene in air, nitrogen, and argon atmospheres studied using femtosecond laser induced breakdown spectroscopy, Spectrochimica Acta Part B-Atomic Spectroscopy, 87 (2013) 121-129.

[38] P. Hough, T. Kelly, C. Fallon, C. McLoughlin, P. Hayden, E. Kennedy, J. Mosnier, S. Harilal, J. Costello, Enhanced shock wave detection sensitivity for laser-produced plasmas in low pressure ambient gases using interferometry, Measurement Science \& Technology, 23 (2012) 125204.

[39] S. Gacek, X. Wang, Dynamics evolution of shock waves in laser-material interaction, Applied Physics a-Materials Science \& Processing, 94 (2009) 675-690.

[40] S.S. Harilal, C.V. Bindhu, M.S. Tillack, F. Najmabadi, A.C. Gaeris, Internal structure and expansion dynamics of laser ablation plumes into ambient gases, Journal of Applied Physics, 93 (2003) 2380-2388.

[41] D. Matthew, M. Morse, Resonant two-photon ionization spectroscopy of jet-cooled UN: Determination of the ground state, Journal of Chemical Physics, 138 (2013) 184303.

[42] J. Wormhoudt, Uranium oxide gaseous ion and neutral infrared spectroscopy, in: Defense Nuclear Agency Technical Report No. DNA 001-02-C-0063, 1983.

[43] L. Kaledin, M. Heaven, Electronic spectroscopy of UO, Journal of Molecular Spectroscopy, 185 (1997) 1-7.

[44] J. Han, V. Goncharov, L. Kaledin, A. Komissarov, M. Heaven, Electronic spectroscopy and ionization potential of UO2 in the gas phase, Journal of Chemical Physics, 120 (2004) 5155-5163.

[45] R.J.M. Konings, T.R. Allen, R.E. Stoller, S. Yamanaka, Comprehensive Nuclear Materials, Elsevier, Amsterdam, Netherlands, 2012.

[46] S.S. Harilal, B.E. Brumfield, M.C. Phillips, Lifecycle of laser-generated air sparks, Physics of Plasmas, 22 (2015) 063301.

[47] H.-J. Kunze, Introduction to plasma spectroscopy, Springer, 2009.

[48] J. Puric, M. Platisa, N. Konjevic, Stark Broadening of Singly Ionized Strontium and Calcium Lines, Z Phys, 247 (1971) 216.

[49] Y. Tian, E.B. Sokolova, R.G. Zheng, Q.L. Ma, Y.P. Chen, J. Yu, Characteristics of the ablation plume induced on glasses for analysis purposes with laser-induced breakdown spectroscopy, Spectrochimica Acta Part B-Atomic Spectroscopy, 114 (2015) 7-14.

[50] M.S. Dawood, A. Hamdan, J. Margot, Axial- and radial-resolved electron density and excitation temperature of aluminum plasma induced by nanosecond laser: Effect of the ambient gas composition and pressure, Aip Adv, 5 (2015) 117136.

[51] C. Aragon, J.A. Aguilera, Characterization of laser induced plasmas by optical emission spectroscopy: A review of experiments and methods, Spectrochimica Acta Part B-Atomic Spectroscopy, 63 (2008) 893-916. 


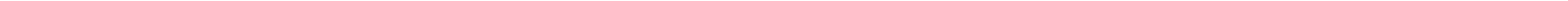

